\title{
Impact of Feng Shui Bedrooms on Self-Assessed Sleep and Well- Being: A Randomized Double-Blind Field Research with Instrumental Biocommunication
}

\author{
Špela Kryžanowski \\ Faculty of Design, Associated Member of the University of Primorska, Slovenia
}

\begin{abstract}
Citation: Kryžanowski Š. Impact of Feng Shui Bedrooms on SelfAssessed Sleep and Well-Being: A Randomized Double-Blin Field Research with Instrumental Biocommunication. SEE Archit Des. 2021 Jun 13; 10057:1-8. http://dx.doi.org/10.3889/seejad.2021.10057

Key words: Architecture; Controlled field research; Doubleblind; Feng shui; Instrumental biocommunication; People's sleep and well-being; Self-assessment

"Correspondence: Špela Kryžanowski, Faculty of Design, Associated Member of the University of Primorska, Slovenia. Email: spela.kryzanowski@fd.si

Received: 06-Apr-2021; Revised: 19-May-2021; Accepted: 21-May-2021; Published: 25-May-2021

Funding: Publication of this article was financially supported by the Scientific Foundation SPIROSKI, Skopje, Republic of Macedonia

Copyright: ๑ 2021 Špela Kryžanowski. This is an open-access article distributed under the terms of the Creative Commons and reproduction in any medium, provided the original author and source are credited.

Competing Interests: The author have declared that no competing interests exist.

Abbreviations: $\mathrm{Cl}$ = confidence intervals; SLEEP = question: "How would you assess the quality of your sleep; WELL-BEING = question: "How would you assess your general well-being?": the moning?"; qEALTH = quetion: "Hlow would you up in your health?"
\end{abstract}

\section{Introduction}

Feng Shui is a traditional Chinese art of designing physical environments, grounded in traditional Chinese philosophy, culture and its historical framework. Throughout the history feng shui was considered as part of traditional Chinese medical treatment and even today some analyse it in the context of traditional Chinese medicine [1], [2], [3]. In the West, feng shui experienced the peak of lay popularity around the millennium's turn, nevertheless, the main stream architectural or any other profession never truly clarified its opinion on the effectiveness of the feng shui phenomenon, especially regarding its impact on one's well-being. As can be concluded from the review of scientific papers, feng shui is being researched through anthropology, traditional and modern urbanism and architecture, sustainability aspect, economy, tourism and health care. There are many fengshui researchers, but taken together, there are only a dozen people committed to checking the effectiveness of fengshui recommendations which promise positive impact on one's well-being. Of course, there are several valid reasons for this. The first problem is the abundance and the variety of fengshui techniques and their variations where there is no single common base upon which the renowned fengshui practitioners would unanimously agree that it represents the corpus of traditional feng shui. If researchers quickly learn 'the fengshui basics' through an uncritical set of popular fengshui manuals, the results of their research cannot be credible, not because of the ineffectiveness of fengshui recommendations but because of incorrect fengshui data input. An additional problem is the original sources written in Chinese characters, in a language that most researchers do not know and therefore they have to depend on indirect translations. In addition, many expressions in Chinese (and also in feng shui) have such a multifaceted and specific 
meaning that there are no corresponding synonyms for them [4], [5]. The ignorance of the cultural-historical framework in which feng shui was created is also an obstacle. How can one consider the high level of subjectivity when using fengshui knowledge in practice? The main problem, however, is the isolation of fengshui effects. How should one design scientific protocol in order to assert that the effects the study identifies are actually the result of the mere performance of good or bad fengshui influences and not other factors? And if this problem is surmounted, how can one take into account the placebo effect?

To solve these problems fengshui researchers apply two approaches: a theoretical approach by comparison with the findings of the modern science without having done actual field research, and the practical approach which involves people (architects or laymen) and analyses of photographs of specific locations. The comparative analyses show [6], [7] that there is a certain similarity between the recommendations of the fengshui school of form and the findings of contemporary environmental psychology. For example: the composition of the five mystical fengshui animals is a symbolical description of a spatial formation where the back of a man or an object is protected, and an open space that can be easily controlled is in front. The practical (or experimental studies) use several approaches. Comparisons with contemporary principles of open spaces or landscape design [8], [9] show, that welldesigned open spaces are also well-designed from the point of view of feng shui. Similar is true for the [10] research, where architects opt to choose a setting for the house which is also good from the point of view of feng shui. Feng shui principles can also be found in modern interior design where [11] discover, that architects incorporate some fengshui recommendations into their bedroom design, albeit unknowingly. So and Lu [12] look for the best position of the bathroom in a typical Hong Kong apartment in relation to computer wind simulation and fengshui recommendations. Another approach is a comparison with the selected sustainable design principles [13] or the analyses, whether fengshui recommendations can be integrated into contemporary models for total building performance [14]. The applicative verification of the effectiveness of the fengshui recommendations was also used in the pilot study of hotel lobbies [15] where a correlation between the economic performance of chosen hotels and a good feng shui of the hotel lobby could be identified. In the analysis of the comfort of medical waiting rooms [16] the area with the best feng shui was also the best from the point of view of the users. The evaluation of feng shui in critical care environment [17] on the other hand, didn't show any statistical significance. It's worth to notice however, that a commercial bagua (the so-called life stations bagua) was used in the study, which according to traditional teachers doesn't even belong to feng shui [18].
From the above examples one can conclude, that some of fengshui recommendations are effective and are already incorporated in the contemporary design practice. But much work still needs to be done. Because this is an important (and still not well enough) researched issue, a research protocol was designed to answer the following question: "Do fengshui recommendations applied on bedrooms have an effect on the quality of sleep and general well-being?".

\section{Material And Methods}

The instrumental biocommunication system Quantec [19], was chosen for the research, because it could:

-implement fengshui recommendations onto the bedrooms without placing too high a burden on participants and

-introduce a control (placebo) group that would allow for a double-blind protocol and thus eliminate the influence of the diverse physical environmental factors and the subjective circumstances of the participants (relationships, family, work, health, etc.).

Quantec is grounded in the assumption of quantum physics that each substance is denoted by a certain oscillation. And that the elemental particles of matter can be expressed as particles or as waves (the double nature of the particles). According to De BroglieBohm's interpretation of quantum mechanics, each particle of a matter is characterized by a certain pilot wave [20]. This wave can propagate in all directions as well as through substances and, as a rule, without the space and time constraints. On the basis of this wave, it should be possible to reconstruct the nature of an object (or person) and its state. Additionally, what feng shui calls qi may be correlated to contemporary research into biofield [21] or orgone [22] and exceeds the limited historical concept of vitalism, where the living force is beyond the research of science. Qi is also being researched scientifically [23], [24], [25]. And recent scientific research claims that the nature of vital force can be attributed to the superfluid vacuum [26]. In practice, Quantec is a diode-based system with a computer program that includes information databases. The diode produces white noise and can show statistically relevant reactions to impulses of group consciousness in cases of high-level mental focus or group emotions [27], [28]. The Quantec database system is pre-made (acupuncture, medicinal herbs, homeopathy) but also open and can be complemented with new databases, the fengshui database. Quantec can thus send information (that is, the fengshui recommendations) in a non-material way onto the chosen photo of the room (building, person, animal, plant etc.) without the need to physically 
rearrange the room. The appropriate information (that is, vibrations in the form of statements, therapeutic information or recommendations that are written in the database) is chosen autonomously by the program and written in the user's information sheet. In the operational phase this information is transmitted (projected through the use of the diode and the program) onto the photo, which is called the process of informing. It can have a single or several cycles, different lengths (a few seconds or minutes) and frequencies (every two hours). Quantec was already used as a research method in other studies [29], [30]. The most recent medical study of Mora et al., [31] shows, that Quantec can have statistically significant effect on cardiovascular risk factors and autonomous modulation of heart rate, whereby Quantec was used only as a one session treatment, lasting for 12 seconds, and using a participant's photograph. In the performed field research one hundred and thirty-four (134) adult persons anonymously responded to the public call for participation and sent a photo of their bed, a sketch of their bedroom and a completed introductory questionnaire with data on sex, age, education, assessment of the quality of their bedroom, the length of their stay in the bedroom and their knowledge of alternative medicine. Each participant received an identification number and was classified into one of three groups: A (mixed group), B (trial group), or C (control - placebo group). The Quantec operator made an information sheet with fengshui recommendations for each participant, based on his/her bedroom photo. We used a new fensghui information database (made by the author) and an existing construction science fengshui database. The new fengshui database drew on prior analyses of fengshui techniques and methodologies. The database contained recommendations of both fengshui schools (the compass school and the school of form) found in English or German literature and used by several traditional fengshui teachers in the same way: Larry Sang [32], Joseph Yu [18] and Eva Wong [33], [34] and in selected sections by Stephen Skinner [35], Derek Walters [36] and Evelin Lip [37].

Quantec sent supportive fengshui recommendations to the participant's bedroom photographs of the B (trial) group during the entire research period (February 19 - June 26), that is, to each participant separately (according to his/her unique information sheet) and in cyclic intervals. Group A (mixed) was under Quantec influence for only the second half of the research period (April 12 - June 26) and group $\mathrm{C}$ acted as control, with information sheets inactivated the whole time. The double-blind protocol was achieved so that only the author knew which participants (labelled with the contact e-mail and an identification number) were in which group and only the Quantec operator knew which group was under which protocol (until the end of the active Quantec phase). The participants had no knowledge about the grouping, and they did not know that the research was about feng shui. They consented to being part of the research, where one would try to improve the ambient quality of their bedroom, by sending positive information using radionics and a computer.

Each participant received a questionnaire approximately every 10 days by e-mail with the following questions:

- How would you assess the quality of your sleep? (hereinafter referred to as the SLEEP)

- How would you assess your general wellbeing? (hereinafter referred to as the WELL-BEING)

- How well rested do you wake up in the morning? (hereinafter referred to as the AWAKENING) and

- How would you assess your health? (hereinafter referred to as the HEALTH). The participants had to answer each question with a value of 1 to 5 (Likert scale from 1 - very bad to 5 - very good). In addition, the participants indicated if some unusual events (positive or negative stress) occurred during the past week and if they spent at least 4 out of 7 nights in the analyzed bedroom. The same fengshui database and a similar concept was previously checked in a pilot study involving 44 participants, lasting for 3 months, and using 6 questions, showing the biggest difference between the fengshui (Quantec) group and the placebo group in the assessment of the quality of sleep. For this reason, the bedroom and the quality of sleep were made the primary research targets.

\section{Results}

The $R$ environment ( $R$ Core Team 2018) was used for statistical data processing. As many as 1340 questionnaires were sent and 1057 (79\%) received. Data received through questionnaires was combined with the data from the introductory questionnaires in a single database. The non-responding participants were not called to respond. In the event of not receiving the answers, the fields were left blank. If the participant gave an intermediate value (3.5), a lowered full value was entered (3.0). There were only a few such instances. Groups A, B and C were equivalent in terms of age, gender, education, knowledge of alternative medicine, assessment of their own bedroom and the presence of stress during the research. The average age of the participants was 39 years. There were significantly more women involved (104) than men (30). The average level of education was graduate level, average self-assessed general knowledge of alternative medicine was 3.05 , the average bedroom rate was 3.5 and participants had lived in their bedrooms on average for almost 9 years, which is a stable period.

Linear mixed models were used to analyse how 
fengshui recommendations chosen for participant's bedrooms (and working through the activation of Quantec information sheet: hereinafter referred to as the feng shui variable) affect the participant's quality of SLEEP, WELL-BEING, AWAKENING and HEALTH. The categorical variable 'group' $(A, B, C)$ was replaced in the model by the numerical variable 'feng shui' (which shows the number of days that elapsed since the activation of Quantec information sheet to the moment when the participant provided the answers; if the participant's information sheet was not activated at the moment of answering, a value 0 was entered). The presence of any extraordinary events (illness, change of work, marriage, promotion, etc.) was included in the model with the descriptive binary variable of stress $(1=$ stress, $0=$ no stress). The model design also considered that each person answered multiple times for the same bedroom and that the responses could depend on the person giving the answer. For that reason, the factor "person" was included in the model as a random influence. Numerical variables for age and prior rating of bedroom were also included in the model, and they were centered to the average value to facilitate the interpretation of the results. The model also included all interactions of the above-mentioned variables (Table 1). In testing multiple hypothesis at the same time (see Tables 2 and 3 ) a $p$-value correction was used that takes into account the interconnectedness of results in the case when several hypotheses are tested on the basis of the same data at the same time. The estimates of the parameters in the linear mixed models were estimated using function Ime from the nlme package in $R$ program [38]. The $p$-values of the effects in the models were corrected for simultaneous hypothesis testing on the basis of multivariate $\mathrm{t}$-distribution using glht function from multcomp package in $\mathrm{R}$ program [39].

Table 1: $P$-values of the variance analysis* for mixed models: SLEEP, WELL-BEING, AWAKENING and HEALTH (without $p$ value correction)

\begin{tabular}{|c|c|c|c|c|}
\hline VARIABILITY SOURCES & $\begin{array}{l}p \text {-value } \\
\text { SLEEP }\end{array}$ & $\begin{array}{c}p \text {-value } \\
\text { WELL-BEING }\end{array}$ & $\begin{array}{c}p \text {-value } \\
\text { AWAKENING }\end{array}$ & $\begin{array}{c}p \text {-value } \\
\text { HEALTH }\end{array}$ \\
\hline stress & $<0.0001$ & $<0.0001$ & $<0.0001$ & $<0.0001$ \\
\hline age cent. ${ }^{*}$ & 0.0043 & 0.0010 & 0.3183 & $<0.0001$ \\
\hline feng shui *** & $<0.0001$ & 0.0010 & 0.0671 & 0.0218 \\
\hline bedroom rating cent. & 0.0025 & 0.1023 & 0.0318 & 0.0074 \\
\hline stress: age cent. & 0.1855 & 0.5741 & 0.9779 & 0.6312 \\
\hline stress: feng shui & 0.2980 & 0.0354 & 0.0830 & 0.5455 \\
\hline age cent.: feng shui & 0.0028 & 0.8646 & 0.0633 & 0.3241 \\
\hline $\begin{array}{l}\text { stress: bedroom rating } \\
\text { cent. }\end{array}$ & 0.1543 & 0.7777 & 0.0042 & 0.0708 \\
\hline $\begin{array}{l}\text { age cent.: bedroom rating } \\
\text { cent. }\end{array}$ & 0.1767 & 0.1992 & 0.0997 & 0.0075 \\
\hline $\begin{array}{l}\text { feng shui: bedroom rating } \\
\text { cent. }\end{array}$ & 0.0090 & 0.0002 & 0.0187 & 0.0775 \\
\hline stress: age cent.: feng shui & 0.6559 & 0.7194 & 0.9497 & 0.0090 \\
\hline $\begin{array}{l}\text { stress: age cent.: bedroom } \\
\text { rating cent. }\end{array}$ & 0.4544 & 0.7664 & 0.1300 & 0.5031 \\
\hline $\begin{array}{l}\text { stress: feng shui: bedroom } \\
\text { rating cent. }\end{array}$ & 0.1763 & 0.0009 & 0.0661 & 0.0061 \\
\hline $\begin{array}{l}\text { age cent.: feng shui: } \\
\text { bedroom rating cent. }\end{array}$ & 0.5499 & 0.7612 & 0.7174 & 0.0989 \\
\hline $\begin{array}{l}\text { stress: age cent.: feng shui: } \\
\text { bedroom rating cent. }\end{array}$ & 0.0035 & 0.0576 & 0.0119 & 0.0320 \\
\hline
\end{tabular}

\section{Question: How Would You Assess the Quality of Your Sleep (SLEEP)?}

In the analysis of the variance of mixed models for the question SLEEP (Table 1) the observed feng shui variable shows a statistically significant impact (at significance level 0.05 ) as a standalone variable and as an interaction of all four variables. In addition, there is a statistically significant interaction of feng shui: bedroom rating and the interaction feng shui: age. These results show that the influence of the variable feng shui is also dependent on the age of the participants and on their assessment of the bedrooms. In Table 2 the mixed model parameters with the corresponding 95\% confidence intervals (hereinafter referred to as $\mathrm{Cl}$ ) are calculated, considering the correction of $p$ values because of the simultaneous hypothesis testing. The influence of the feng shui (with constant values of other variables in the model) remains positive and statistically significant. For each day of the Quantec activation the value increases by $0.003(95 \% \mathrm{Cl} 0.001,0.006)$. The bedroom rating variable has a positive significant impact and the stress and age negative.

Table 2: Parameter estimates and their $95 \% \mathrm{Cl}$ for mixed model for SLEEP (with $p$-value correction) *

\begin{tabular}{lcccc}
\hline SLEEP & Estimate & Lower & Uper & $p$-value \\
\hline Model parameters & & $\mathrm{Cl} 95-\%$ & $\mathrm{Cl} 95-\%$ & \\
Intercept** & 3.629 & 3.470 & 3.789 & \\
stress & -0.2423 & -0.4295 & -0.0552 & 0.00225 \\
age cent. & -0.0154 & -0.0269 & -0.0040 & 0.00117 \\
feng shui & 0.0034 & 0.0010 & 0.0058 & $<0.001$ \\
bedroom rating cent. & 0.2515 & 0.0532 & 0.4498 & 0.00307 \\
age cent.: feng shui & 0.0002 & -0.0000 & 0.0004 & 0.07674 \\
feng shui: bedroom rating cent. & -0.0029 & -0.0058 & 0.0000 & 0.06099 \\
stress: age cent.: feng shui: bedroom & 0.0005 & 0.0000 & 0.0009 & 0.04860 \\
rating cent. & & & & \\
\hline Legend: ${ }^{*}$ Only significant and marginally significant results are shown; & ** The estimate for the \\
intersection 3.63 (95\% Cl 3.47, 3.79) is the average SLEEP estimate in the stress-free group at the \\
mean age, the average bedroom rating and the time from activation (feng shui variable) at value 0;
\end{tabular}
mean age, the average bedroom rating and the time from activation (feng shui variable at value Bold script indicates statistical significance at significance level 0.05 .

What does this result mean? It means that fengshui recommendations sent onto bedroom photographs had a significant effect on participant's self-assessment of the quality of SLEEP. The impact of the fengshui variable is highly statistically significant $(p$ $<0.001$ ). Although an increase of 0.003 per day, from the point of view of the value itself, is irrelevant, it is important, because the impact becomes interesting and noticeable over a longer period of time. Within 10 days, the rise in the value of quality of sleep due to the fengshui support increases to 0.03 , and within 100 days, to 0.30 . The research lasted about 120 days and the average value of the estimated SLEEP quality in people without stress could rise from 3.63 (intercept estimate) to 3.99 , i.e., cumulatively by noticeable 0.36 . This is comparable to the positive impact of the bedroom rating 0.25 (where the increase of value is connected with an increase of bedroom rating by 1 point). Figure 1 additionally shows that stress diminishes the positive impact of fengshui recommendations on SLEEP. 


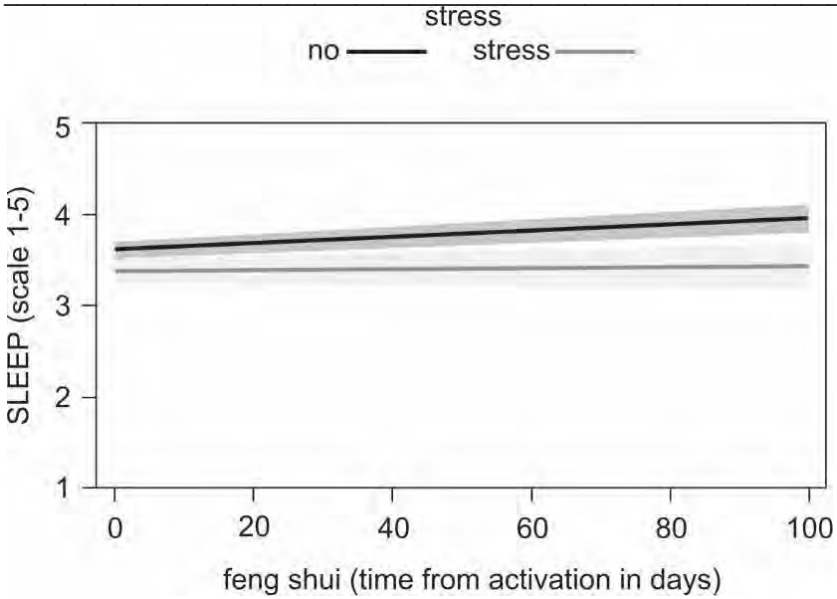

Figure 1: Model predicted average SLEEP estimate with corresponding $95 \% \mathrm{Cl}^{*}$; Legend: * dependant on feng shui and stress variables (at average age and average bedroom rating)

The prior bedroom rating also seems to be an important space variable (increase of value by 0.25 , with $95 \% \mathrm{Cl} 0.05,0.45)$ : the higher the bedroom rating, the higher the quality of SLEEP (see Figure 2). Furthermore, the interaction of the bedroom rating and feng shui (which is marginally statistically significant at $p=0.06$ ) indicates, that the influence of fengshui recommendations should also be explained in dependence with the self-assessment of the quality of the space in which a person sleeps. Similar trend is reflected by the answers for the question about WELLBEING.

stress
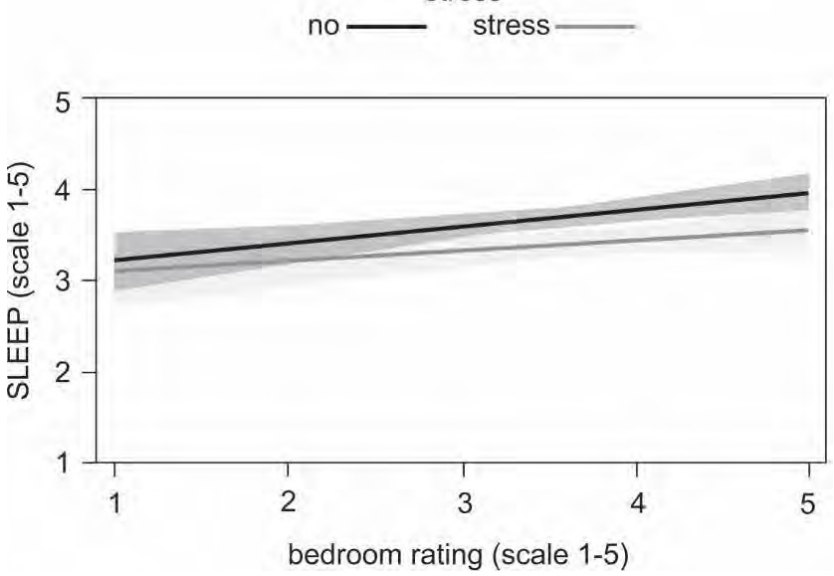

Figure 2: Model predicted average SLEEP estimate with corresponding $95 \% \mathrm{Cl}^{*}$; Legend: * dependant on bedroom rating and stress variables (at average time from activation of Quantec (feng shui variable) and average age)

\section{Question: How Would You Assess Your General Well-Being (WELL-BEING)?}

The impact of fengshui recommendations on bedrooms can also be noticed through the participant's self- assessment of their general well-being. Statistical significance of the independent variable feng shui in the Table $1,(p$-value before correction $=0.001)$ is lost after $p$ - correction (see Table 3). But the interaction of feng shui and bedroom rating variable remains highly significant $\quad(p<0.001,95 \% \quad \mathrm{Cl} \quad-0.007,-0.001)$. Additionally, there is a marginal statistical significance ( $p=0.06)$ for the interaction of variables stress: feng shui: bedroom rating.

Table 3: Parameter estimates and their $95 \% \mathrm{Cl}$ for mixed model for WELL-BEING (with p-value correction) *

\begin{tabular}{lcccc}
\hline $\begin{array}{l}\text { WELL-BEING } \\
\text { Model parameters }\end{array}$ & Estimate & $\begin{array}{c}\text { Lower } \\
\text { Cl 95-\% }\end{array}$ & $\begin{array}{c}\text { Uper } \\
\text { Cl 95-\% }\end{array}$ & p-value \\
\hline Intercept & 3.785 & 3.650 & 3.919 & \\
stress & -0.4994 & -0.6796 & -0.3192 & $<0.001$ \\
age cent. & -0.0103 & -0.0199 & -0.0007 & 0.0244 \\
feng shui: bedroom rating cent. & -0.0042 & -0.0070 & -0.0014 & $<0.001$ \\
stress: feng shui: bedroom rating & 0.0052 & -0.0001 & 0.0106 & 0.0604 \\
cent. & \multicolumn{4}{l}{} \\
\hline Legend: * Only significant and marginally significant results are shown; Bold script indicates
\end{tabular}
statistical significance at significance level 0.05

To better understand the negative impact of interaction feng shui: bedroom rating Figure 3 was prepared.

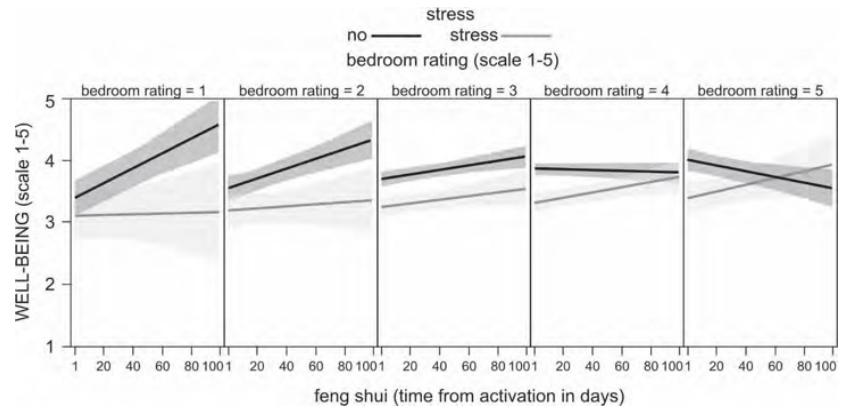

Figure 3: Model predicted average WELL-BEING estimate with corresponding $95 \% \mathrm{Cl}^{*}$ Legend: * dependant on bedroom rating, feng shui and stress variables (at average age)

The model prediction shows that the impact of fengshui variable on WELL-BEING differs. For people without stress, as the value of the bedroom rating increases, the positive impact of the fengshui variable decreases. For a low bedroom rating (grade 1 or 2 ), the positive impact of feng shui for unstressed persons is the highest. With a medium and better bedroom rating (grades 3 and 4), the impact of feng shui is small. At the highest value of the bedroom rating (rating 5), the impact of feng shui on people without stress turns negative. This can be explained by the fact that the fengshui recommendations on the WELL-BEING parameter work primarily with poor bedroom ratings (the settings people are not satisfied with), which would also be some general expectation. Where, at least from the standpoint of one's own assessment, the bedroom is almost ideal, this factor does not work, or even slightly 'disturbs' WELL-BEING, presumably because the general fengshui recommendations negatively interfere with the very concrete optimally functioning factors of a realistic bedroom layout. And the exact opposite is true for people under stress. Stress at low bedroom ratings seams to inhibit the positive influence 
of fengshui recommendations on well-being. At higher bedroom ratings people under stress seam to benefit most from the fengshui support, as if the physically well-designed environments could help overpass the blockage that inhibited the receipt of fengshui support at lower bedroom ratings.

\section{Questions on Awakening and Health}

For question "How Well Rested Do You Wake Up in the Morning?" (AWAKENING) and "How would you assess your health?" (HEALTH) the variable feng shui after the $p$-value correction does not appear statistically significant (neither as a standalone variable or in interaction with other variables). The effects on AWAKENING can best be explained by the significant variables stress (negative impact), bedroom rating (positive impact) and an interaction of the two. The effects on HEALTH are best explained by the significant variables stress and age (both negative impact) and the interaction of age and bedroom rating (positive impact).

Although insignificant, the model prediction for interaction of feng shui and a bedroom rating estimate for HEALTH (Figure 4) shows a similar trend to that of highly significant WELL-BEING (Figure 3). The positive impact of variable feng shui for non-stressed persons on HEALTH is seen only in low bedroom ratings (grades 1 and 2), and the highest rating (5) makes the impact of feng shui in non-stressed persons negative. And conversely, for people under stress at low bedroom ratings the negative impact of feng shui support increases over time and for stressed people in well-rated bedrooms the impact of feng shui increases in a positive way over time. It looks like that in poorly rated environments feng shui has a positive effect on health of people without stress, and in well-rated environments feng shui has a positive effect on people under stress.

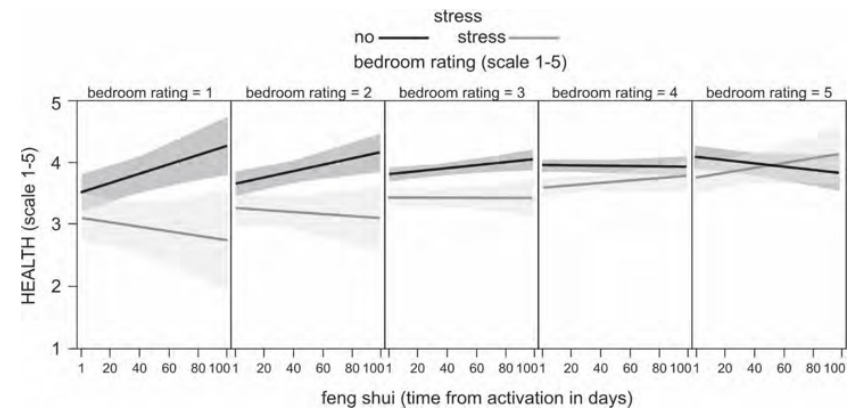

Figure 4: Model predicted average HEALTH estimate with corresponding $95 \% \mathrm{Cl}^{*}$ Legend: ${ }^{*}$ dependant on bedroom rating, feng shui and stress variables (at average age)

\section{Discussion}

The results are important because they identify a new field of impact on one's well-being generated by physical space. Among all fengshui field studies identified (and presented in the introduction), there is not one that covers such a large sample of physical spaces and is at the same time able to isolate or expose the actual effects of fengshui recommendations on user's self-assessed feelings. The results also suggest that the impact of fengshui recommendations is most noticeable when used in a very focused way. Feng shui was most significant for SLEEP and participants received fengshui support only when they were actually staying in bed, which is probably why they linked it directly to a higher quality of SLEEP. At least two other feng shui studies focus on bedrooms, but none tried to measure the actual effects on users of fengshui recommendations. Our results also suggest that the impact of fengshui recommendations is not linear or straightforward, as one might expect. On the contrary, it seems to be dynamic and in a direct correlation with the level of our living environment satisfaction and our exposure to stress. The results expose the importance of physical space factors, either through fengshui or through bedroom rating variable (or their interactions). If the statistical significance of fengshui influence is not yet common, the positive impact of a well-designed environment on human performance has been repeatedly confirmed through research in environmental psychology [40]. The shown effectiveness of remote transmission of information is also important. Not only is it possible to influence the quality of sleep and well-being of persons in the room through the use of fengshui recommendations, but it is obviously enough to transmit them on a purely informational level (that is, at the energy-vibrational or quantum vacuum level, without physical interventions). This can be positive news for all those space situations where physical remodelling is impossible for one reason or another: finance, space size, construction limitations, etc.

Western architects and part of scientists still perceive feng shui as a pseudoscience and this empirical account of its impact (though on a small scale) is an important encouragement for further scientific research, that could allow for controlled integration of selected fengshui recommendations into the part of contemporary architecture and science, that specializes in the quality of living.

To better understand the possibilities and limitations of presented fengshui research protocol, further research is necessary. One issue is the optimal length of the time intervals (during which the fengshui information is sent) that bring the best results. Another issue is which of the fengshui schools (school of form or compass school) and various techniques can show the best positive impact? Can statistically significant 
effects using feng shui be obtained also in other areas of life (informing workspace for a better work environment)? How can a correlation between feng shui, stress and satisfaction with existing living environment be better understood?

\section{Conclusions}

Results suggest that:

- fengshui recommendations can have a highly statistically significant impact on user's self-assessed feelings. The impact in the study is most obvious for SLEEP and WELL-BEING

- the impact of fengshui recommendations is dynamic and it is in correlation with the level of participant's living environment satisfaction and stress

- it is possible to remotely transmit fengshui recommendations. This is important in all situations where physical remodelling is impossible.

Notwithstanding the many open questions, the research carried out represents an important contribution to experimental evaluation of fengshui recommendations and their impact on self-assessed well-being. It shows that it is possible to use a treatment - control protocol with feng shui. Which further suggests that it is possible to develop a systematic empirical methodology for the research of effectiveness of fengshui recommendations in general, or specifically for selected fengshui school or method. Furthermore, the research is important because it represents an introduction to exploring the potential of remote informing of the physical space which goes well beyond the realm of feng shui.

\section{References}

1. Gulmen FM. Energy medicine. The American journal of Chinese medicine. 2004; 32(05):651-8.

2. Clarke DB, Doel MA, Segrott J. No alternative? The regulation and professionalization of complementary and alternative medicine in the United Kingdom. Health \& Place. 2004; 10(4):329-38.

3. Matthews MR. Feng Shui: teaching about science and pseudoscience. Springer International Publishing; 2019.

4. Gillespie AR. A source book in Chinese philosophy. Greenwood Publishing Group; 1963.

5. Mak MY, So AT. Scientific feng shui for the built environment: Fundamentals and case studies. City University of Hong Kong Press; 2011.

6. Xu J. A framework for site analysis with emphasis on Feng Shui and contemporary environmental design principles (Doctoral dissertation, Virginia Tech). 2003.

7. Bonaiuto M, Bilotta E, Stolfa A. "Feng Shui" And Environmental Psychology: A Critical Comparison. Journal of Architectural and planning research. 2010:23-34.

8. Ke-Tsung $H$, Sinha A. An empirical study of feng-shui in landscape. Environments. 1996; 23(3):36.

9. Lynch ES. Feng Shui as a site design tool: Assessing conditions of human comfort in urban places. 2003.

10. Mak MY, Ng ST. The art and science of Feng Shui-a study on architects' perception. Building and Environment. 2005; 40(3):427-34.

11. Hong WT, Abdul-Rahman H, Wang C. Are Feng Shui Bedroom Rules Practical from the Architectural Perspective? Proceedings of the Academic Journal of Feng Shui. 1st SymposiumOceania. Sydney: Oceania University of Technology. 2017.

12. So AT, Lu JW. Natural ventilation design by computational fluid dynamics - a feng-shui approach. Architectural science review. 2001; 44(1):61-9.

13. Mak MY. An empirical study of modern sustainable o ce buildings in Sydney from the feng shui perspective. Proceedings of the Academic Journal of Feng Shui. 1st Symposium-Oceania. Sydney: Oceania University of Technology. 2017.

14. Pheng LS, Xiaopeng D, Ting QL. Assimilating total building performance mandates with Chinese geomancy principles and scenarios. Facilities. 2012; 30(13/14):558-589.

15. Poulston J, Bennett R. Feng shui, fact, and fiction: An exploratory study. Facilities. 2012; 30(1):2.

16. Bazley C, Vink P, Montgomery J, Hedge A. Interior effects on comfort in healthcare waiting areas. Work. 2016; 54(4):791-806.

17. Charles R, Glover S, Bauchmüller K, Wood $D$. Feng shui and emotional response in the critical care environment (FARCE) study. Anaesthesia. 2017; 72(12):1528-31.

18. Moran E, Yu MJ, Biktashev V. The complete Idiot's guide to Feng Shui. New York: Alpha books; 2005 Mar 1.

19. Von Buengner P. Instrumentalle Biokommunikation mit QUANTEC. Altkirchen: M-TEC Verlag; 2007.

20. Tiller WA. Psychoenergetic science: a second Copernican-scale revolution. Pavior Pub; 2007. 
21. Zerovnik E, Markič O, Ule A. Philosophical Insights about Modern Science. Nova Science Pub Incorporated; 2009.

22. De Meo, J. The Orgone Accumulator Handbook: Wilhelm Reich's Life Energy Discoveries and Healing Tools for the 21st Century With Construction Plan. Greensprings: Natural Energy Works; 2010.

23. Yan $X$, Lu F, Jiang $H$, Wu X, Cao W, Xia Z, Shen $H$, WANG J, DAO M, LIN H, ZHU R. Certain physical manifestation and effects of external qi of Yan Xin Life Science Technology. Journal of Scientific Exploration. 2002; 16(3):381-411.

24. Yan $X$, Shen $H$, Jiang $H$, Zhang $C$, Hu D, Wang J, Wu X. External Qi of Yan Xin Qigong differentially regulates the Akt and extracellular signalregulated kinase pathways and is cytotoxic to cancer cells but not to normal cells. The international journal of biochemistry \& cell biology. 2006; 38(12):2102-13.

25. Lu Z. Scientific Qigong exploration: the wonders and mysteries of Qi. Amber Leaf Press; 1997.

26. Meijer DK, Geesink JH. Phonon Guided Biology. Architecture of Life and Conscious Perception Are Mediated by Toroidal Coupling of Phonon, Photon and Electron Information Fluxes at Discrete Eigenfrequencies. NeuroQuantology. 2016; 14(4):718755.

27. Roger Nelson. The Global Consciousness Project [Internet]. Roger Nelson; 1999-2020. Available from: https://noosphere.princeton.edu/.

28. Nelson RD, Bradish GJ, Dobyns YH, Dunne BJ, Jahn RG. FieldREG anomalies in group situations. Journal of Scientific Exploration. 1996; 10(1):111-41.

29. Schneider R, Walach $H$. Randomized
Double-Blind Pilot Study on Psychological Effects of a Treatment with 'Instrumental Biocommunication'. Complementary Medicine Research. 2006; 13(1):3540.

30. Jobst $D$. Practical study on the therapy of pollinosis with the radionics system QUANTEC ${ }^{2}$. 2016.

31. Mora JA, Chagas EF, Camilo GF. Acute effect of Quantec therapy on cardiovascular risk factors and autonomous modulation of heart rate. Int $\mathrm{J}$ Complement Alt Med. 2018; 11(3):149-53.

32. Sang L. The principles of feng shui. American Feng Shui Institute; 2004.

33. Wong E. Feng-shui: the ancient wisdom of harmonious living for modern times. Shambhala Publications; 1996.

34. Wong $E$. A master course in feng-shui. Boston: Shambhala; 2001.

35. Skinner, S. The living earth manual of fengshui: Chinese geomancy. London: Routledge \& Kegan Paul; 1976.

36. Volters, D. Feng Shui. Beograd: Esotheria; 1998.

37. Lip E. Feng Shui for Bussiness. Singapore: Times Books International; 1994.

38. Team RC. R: a language and environment for statistical computing. R Foundation for Statistical Computing, Vienna. http s. www. R-proje ct. org. 2018.

39. Bretz F, Hothorn T, Westfall P. Multiple comparisons using R. CRC Press; 2010.

40. Augustin S, Frankel N, Coleman C. Place advantage: Applied psychology for interior architecture. John Wiley \& Sons; 2009 Apr 6. 